\title{
Improved antitumor effect of ionizing radiation in combination with rapamycin for treating nasopharyngeal carcinoma
}

\author{
DI WANG $^{1 *}$, LICHEN GAO $^{2 *}$, XUETING LIU $^{3}$, CHUANG YUAN $^{3}$ and GUIHUA WANG ${ }^{1}$ \\ Departments of ${ }^{1}$ Oncology and ${ }^{2}$ Pharmacy, Changsha Central Hospital; ${ }^{3}$ Medical Research \\ Center, Changsha Central Hospital, Changsha, Hunan 410004, P.R. China
}

Received August 14, 2015; Accepted February 17, 2017

DOI: $10.3892 / \mathrm{ol} .2017 .6208$

\begin{abstract}
The aim of the present study is to investigate if rapamycin is a radiosensitizer of nasopharyngeal carcinoma (NPC), and to identify which pathways are involved in radiation sensitization. In vitro, using untreated cells as the control, NPC cells were treated with rapamycin, ionizing radiation (IR) or both. Differences in the phosphorylation of ribosomal protein S6 and glycogen synthase kinase (GSK) 3 $\beta$, expression of cyclin D1, clonogenic survival, number of phosphorylated histone subunit $2 \mathrm{AX}(\gamma \mathrm{H} 2 \mathrm{AX})$ foci, and cell cycle status between the study groups were compared. The results indicated that rapamycin alone decreased the phosphorylation of S6 and GSK3 $\beta$, as well as the expression of cyclin D1, in NPC cells. Thus, rapamycin-treated NPC cells had lower cell viability, and higher DNA damage and G1 arrest than control cells. In addition, the combination of rapamycin and IR caused the highest cell death, DNA damage and G1 arrest when compared with the effects caused by either treatment alone. In conclusion, rapamycin improves the anti-tumor effect of IR for treating NPC through inhibiting the Akt/mechanistic target of rapamycin/S6 and Akt/GSK3ß/cyclin D1 signaling pathways.
\end{abstract}

\section{Introduction}

Nasopharyngeal carcinoma (NPC) is a common malignant head and neck cancer in Southeast Asia and Southern China, with an incidence rate of $>20$ per 100,000 people per year

Correspondence to: Dr Guihua Wang, Department of Oncology, Changsha Central Hospital, 161 Shaoshan Southern Road, Changsha, Hunan 410004, P.R. China

E-mail: wangguihua5708@163.com

Dr Chuang Yuan, Medical Research Center, Changsha Central Hospital, 161 Shaoshan Southern Road, Changsha, Hunan 410004, P.R. China

E-mail: aeolus.yc@connect.polyu.hk

*Contributed equally

Key words: rapamycin, nasopharyngeal carcinoma, radiosensitivity, Akt-associated pathways (nearly 100-fold higher than the rate in Western countries) (1). Fractionated radiotherapy (RT) with a fraction dose of 2 Gy to a total dose of 60-70 Gy is a common/standard strategy for treating NPC due to its advantages in the preservation of normal tissues $(2,3)$. However, surviving tumor cells following fractionated RT may be radioresistant, particularly in patients with advanced NPC, and this cell population contributes to the majority of RT failures (4). Thus, improving radiation sensitization in the treatment of NPC remains a challenge.

It has been reported that the phosphatidylinositol-4, 5-bisphosphate 3-kinase (PI3K)/Akt signaling pathway regulates cellular functions involving mechanisms of radioresistance in head and neck cancer, including intrinsic radioresistance, tumor cell proliferation and hypoxia (5). Shimura (6) previously overviewed the molecular mechanisms of cancer radioresistance acquirement during fractionated RT. It is assumed that fractionated RT damages the DNA of cancer cells and induces DNA double strand breaks (DSBs), which subsequently activate DNA-dependent protein kinase (PK) and then Akt. Activated Akt induces the phosphorylation of glycogen synthase kinase (GSK) $3 \beta$ at serine 9 , which decreases cyclin D1 proteolysis. As a consequence, cyclin D1 accumulates in the nucleus of cells in $\mathrm{S}$ phase, thus perturbing DNA replication, triggering the DNA damage checkpoint and mediating more DNA DSBs. DNA DSBs in turn induce the activation of DNA-PK and eventually mediate cyclin D1 overexpression, which leads to the proliferation and progression of cancer cells and eventually to radioresistance (6).

Mammalian target of rapamycin (mTOR), which is activated by phosphorylated $\mathrm{Akt}$, is a core regulator of protein translation (7). It has been reported that the Akt/mTOR signaling pathway is also responsible for cancer radioresistance (8-10). Additionally, mTOR can decrease the kinase activity of GSK3 $\beta$, responsible for the degradation of cyclin D1 (11). Previous studies have shown that mTOR inhibition improves the radiosensitivity of prostate, breast, bladder and brain cancer (12-15).

Rapamycin, an important inhibitor of mTOR and a drug used for suppressing autoimmune responses in organ transplantation (16), has been reported to act as a radiosensitizer in the RT treatment of prostate, breast, bladder and brain cancer (12-15). However, the anti-tumor effects of the combination of rapamycin and ionizing radiation (IR) for treating NPC are still unknown. In the present study, it was hypothesized that 
rapamycin could overcome the radioresistance of NPC, and that the presence of rapamycin could improve the anti-tumor effects of IR in the treatment of NPC. Thus, in the present study, the effect of the combination of rapamycin and IR on the DNA damage and cycle status of NPC cells was investigated using an in vitro model of NPC.

\section{Materials and methods}

Cell culture and viability assays. HNE1 NPC cells were obtained from the Cancer Research Institute of Central South University (Changsha, China). Cells were cultured in RPMI medium (Thermo Fisher Scientific, Inc., Waltham, MA, USA) containing fetal bovine serum (10\% v/v, Thermo Fisher Scientific, Inc.), L-glutamine $(2 \mathrm{mM})$, penicillin $(100 \mathrm{U} / \mathrm{ml})$ and streptomycin $(100 \mu \mathrm{g} / \mathrm{ml})$, and maintained at $37^{\circ} \mathrm{C}$ and $5 \% \mathrm{CO}_{2}$. Cell viability was assessed using an MTT assay according to the manufacturer's protocol (Sigma-Aldrich; Merck KGaA, Darmstadt, Germany).

Western blotting. Cells were treated with rapamycin, IR or a combination of both, while untreated cells served as the control. Following IR treatment for $24 \mathrm{~h}$, cells were scraped on ice and re-suspended for $30 \mathrm{~min}$ at $4^{\circ} \mathrm{C}$ in cold radioimmunoprecipitation assay lysis buffer containing a cocktail of phosphatase and protease inhibitors (Roche Diagnostics, Indianapolis, IN, USA). Clear lysates were obtained upon centrifugation at $4^{\circ} \mathrm{C}$ and $10,000 \times \mathrm{g}$ for $10 \mathrm{~min}$. The protein concentration of the lysate was measured using Pierce BCA Protein Assay kit (Thermo Fisher Scientific, Inc.). Protein samples $(50 \mu \mathrm{g})$ were loaded onto $10 \%$ polyacrylamide gels, and electrophoresis was subsequently performed. The proteins in the gels were then transferred to polyvinylidene fluoride membranes at $100 \mathrm{~V}$ for $90 \mathrm{~min}$. The membranes were blocked with a phosphate buffer saline (PBS) solution containing 5\% skimmed milk or $5 \%$ bovine serum albumin at $37^{\circ} \mathrm{C}$ for $1 \mathrm{~h}$, and then western blotted with the following rabbit anti-human monoclonal primary antibodies at $4^{\circ} \mathrm{C}$ overnight: Anti-phosphorylated Akt (Ser473 phosphorylation, cat. no. \#4046), anti-total Akt (cat. no. \#4685), anti-phosphorylated GSK3 $\beta$ (Ser9 phosphorylation, cat. no. \#5558), anti-total GSK3 $\beta$ (cat. no. \#12456), anti-phosphorylated S6 (Ser240/244 phosphorylation, cat. no. \#5364), anti-total S6 (cat. no. \#2217), anti-cyclin D1 (cat. no. \#2978) and anti- $\beta$-actin (cat. no. \#4970). All primary antibodies were purchased from Cell Signaling Technology, Inc. (Danvers, MA, USA) and diluted to 1:1,000. Upon washing five times with PBST (PBS containing 0.1\% Tween-20), the membranes were incubated with the appropriate anti-rabbit secondary antibodies (dilution, 1:2,000; cat. no. \#7074; Cell Signaling Technology, Inc.) at $37^{\circ} \mathrm{C}$ for $2 \mathrm{~h}$. Following five washes in PBST, an enhanced chemiluminescence detection system (GE Healthcare Life Sciences, Chalfont, UK) was used to reveal the protein bands of interest on X-ray films (Kodak, Rochester, New York, USA). The films were scanned using an SCX-4321NS imaging system (Samsung Group, Seoul, Korea), and the protein levels were normalized to $\beta$-actin.

Analysis of phosphorylated histone subunit $2 A X(\gamma H 2 A X)$ foci. Residual DNA damage in irradiated or non-irradiated HNE1 cells was assessed by measuring residual $\gamma \mathrm{H} 2 \mathrm{AX}$
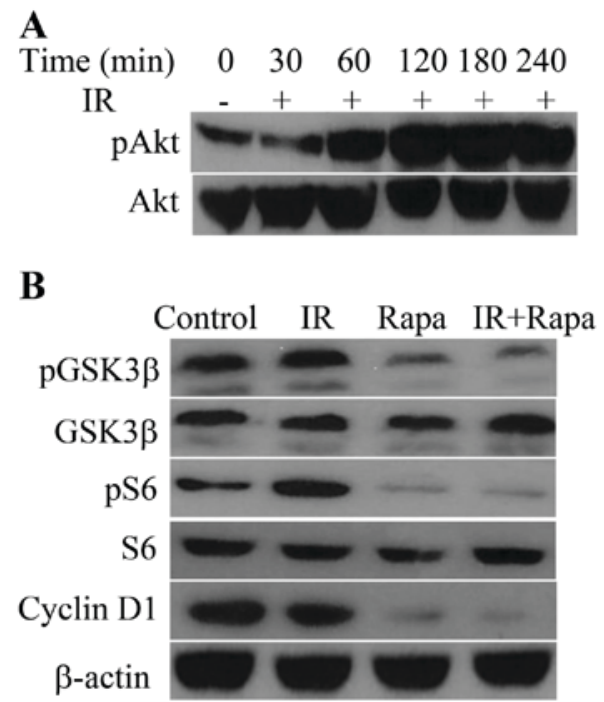

Figure 1. Effects of IR or/and rapamycin on Akt-associated pathways (A) Phosphorylation of Akt in HNE1 cells following treatment with a radiation dose of 4 Gy for 0, 30, 60, 120, 180 and 240 min. (B) Phosphorylation of GSK $3 \beta$ and ribosomal protein S6, and expression of GSK3 $\beta$, S6, cyclin D1 and $\beta$-actin, in HNE1 cells treated with a radiation dose of $4 \mathrm{~Gy}, 100 \mathrm{nM}$ rapamycin or both. Untreated HNE1 cells served as the control. IR, ionizing radiation; Rapa, rapamycin; GSK, glycogen synthase kinase; p, phosphorylated.

foci. Cells were treated with rapamycin $(100 \mathrm{nmol} / \mathrm{l})$ for $10 \mathrm{~h}$, followed by treatment with or without $4 \mathrm{~Gy}$ of irradiation, and then cultured for $24 \mathrm{~h}$. Subsequently, the number of residual $\gamma \mathrm{H} 2 \mathrm{AX}$ foci per cell was counted in five random visual fields under with a DM 2000 LED microscope (Leica Microsystems GmbH, Wetzlar, Germany) (17).

Cell cycle assay. HNE1 cells were plated onto T25 tissue culture flasks (Falcon; Corning Incorporated, Corning, NY, USA) and incubated overnight to allow cells to reach mid-log growth phase. Tumor cells were treated with rapamycin $(100 \mathrm{nmol} / \mathrm{l})$ for $10 \mathrm{~h}$ prior to irradiation and cultured for $24 \mathrm{~h}$ post-irradiation. All adherent cells were suspended using $0.5 \%$ trypsin/EDTA (Thermo Fisher Scientific, Inc.) and harvested upon washing with PBS. Following another wash with ice-cold $70 \%$ ethanol, the cells were incubated with a mixture of $200 \mu \mathrm{g} / \mathrm{ml} \mathrm{RNaseA}$ with $50 \mu \mathrm{g} / \mathrm{ml}$ propidium iodide in a dark room for $30 \mathrm{~min}$ at room temperature. Cell cycle status was examined using flow cytometry (BD Accuri ${ }^{\mathrm{TM}}$; BD Biosciences, Franklin Lakes, NJ, USA). Data were representative of three independent experiments.

Statistical analysis. All statistical analyses were performed using the SPSS 12.0 software package (SPSS, Inc., Chicago, IL, USA). Differences between groups were compared using analysis of variance and then a post-hoc test. All tests were two-tailed and $\mathrm{P}<0.05$ was considered to indicate a statistically significant difference.

\section{Results}

Protein expression and phosphorylation in HNE1 cells following various treatments. Upon exposure to radiation, 

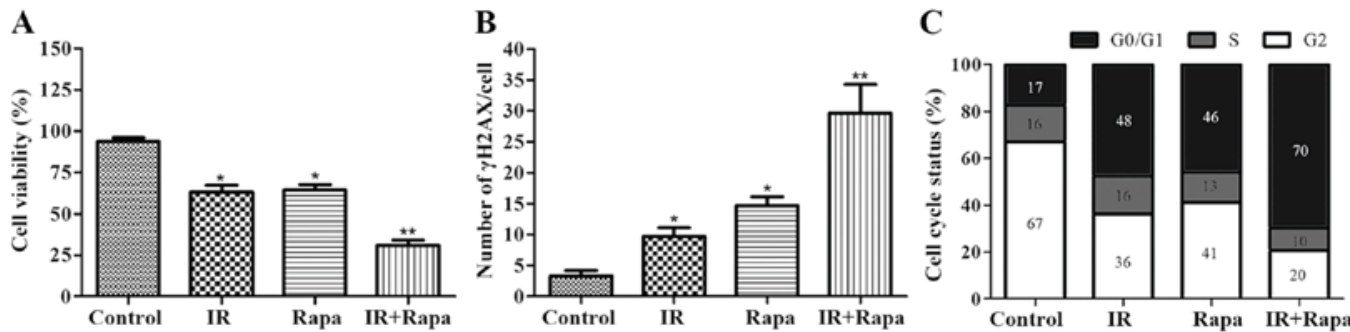

Figure 2. Effects of IR or/and rapamycin on HNE1 cells in an in vitro model for NPC. HNE1 cells were untreated, or treated with a radiation dose of 4 Gy, $100 \mathrm{nM}$ rapamycin or both. (A) Cell viability, (B) DNA damage and (C) cell cycle status were evaluated. ${ }^{*} \mathrm{P}<0.05$ compared with the control group, ${ }^{* *} \mathrm{P}<0.05$ compared with the groups treated either with IR or rapamycin. IR, ionizing radiation; Rapa, rapamycin; $\gamma \mathrm{H} 2 \mathrm{AX}$, phosphorylated histone subunit $2 \mathrm{AX}$.

the phosphorylation of Akt (Ser473) in HNE1 cells increased in a time-dependent manner and peaked at $180 \mathrm{~min}$ post-IR (Fig. 1A). The phosphorylation of GSK3 $\beta$, one of the downstream targets of Akt (6), was retarded in the presence of rapamycin, which resulted in the decrease of cyclin D1 levels (Fig. 1B). In addition, rapamycin targeted S6, a downstream target of mTOR (7), and decreased the phosphorylation of S6 in irradiated HNE1 cells (Fig. 1B).

Cell viability, DNA damage and cell cycle status in HNE1 cells following various treatments. Cell viability was significantly lower, and the number of residual $\gamma \mathrm{H} 2 \mathrm{AX}$ foci was significantly higher, in the groups subjected to treatment with IR (4 Gy) and/or rapamycin (100 nmol/l) than in the untreated control group (Fig. 2A). Furthermore, as compared with cells treated with IR alone, lower cell viability and a higher number of residual $\gamma \mathrm{H} 2 \mathrm{AX}$ foci were detected in cells treated with both rapamycin and IR (Fig. 2B). For cell cycle status analysis, treatment with both rapamycin and IR led to an increase in the number of cells in G1 phase and a decrease in the number of cells in S phase compared with cells treated with either IR or rapamycin alone, thus indicating an effect of rapamycin on G1 arrest (Fig. 2C).

\section{Discussion}

Although fractionated RT can treat the majority of NPC patients, the efficacy of RT is limited in certain cases such as advanced and recurrent NPC $(3,18)$. Furthermore, fractionated RT may increase the radioresistance of surviving cancer cells $(6,9,19,20)$. It has been demonstrated that the activation of the PI3K/Akt signaling pathway serves a vital role in the radioresistance of head and neck cancer (5). IR induces DNA DSBs, which consequently activate the PI3K/Akt signaling pathway for DNA DSB repair (6). This phenomenon was reproduced in the present study, in which the phosphorylation of Akt at Ser473 in HNE1 cells was increased in a time-dependent manner following exposure to a dose of $4 \mathrm{~Gy}$ of IR. Once the PI3K/Akt signaling pathway is activated, downstream cascades, including the Akt/mTOR and Akt/GSK3 $\beta /$ cyclin D1 signaling pathways, are triggered, thus increasing the growth, proliferation, survival and motility of cancer cells (5), which eventually facilitates radioresistance acquirement in head and neck cancer (21).

Rapamycin is an inhibitor of mTOR, and can prevent the phosphorylation of GSK $3 \beta$ at serine 9 to consequently increase cyclin D1 proteolysis (11). Previous studies have shown that inhibition of these Akt-associated pro-survival signaling pathways enhances the radiosensitivity to RT of various cancers (12-15). This improvement may be due to, firstly, the antifungal characteristics of rapamycin itself, and secondly, to the excessive autophagy induced by rapamycin (15). In addition, Liu et al (22) demonstrated that leukemia inhibitory factor (LIF) activated p70 S6 kinase (p70S6K) and its downstream molecules, GSK3 $\alpha / \beta$, thus leading to the proliferation of NPC cells. It is well known that mTOR is the upstream regulator of p70S6K (7). In the study by Liu et al (22), mTOR inhibitors, including rapamycin and everolimus, significantly attenuated the LIF-induced activation of p70S6K and consequently suppressed the proliferation of NPC cells. A consistent result was observed in the present study, since rapamycin alone significantly suppressed the viability and progression of NPC, and improved the anti-tumor effects of IR in the treatment of NPC. The present study demonstrated that rapamycin decreased the phosphorylation of S6 [a downstream target of mTOR (7)], GSK3 $\beta$ and cyclin D1 [a downstream target of GSK3 $\beta(6)]$ in HNE1 cells.

S6, which is activated by phosphorylated p70S6K (a downstream target of mTOR), is a ribosomal protein and a crucial component of the translation machinery of messenger RNA (23). Reduction of activated S6 decreases protein synthesis and cell growth, and prolongs the G1 phase of the cell cycle (24). Cyclin D1 is an indispensible factor in the transition from $\mathrm{G} 1$ to $S$ phase (25). Additionally, cyclin D1 serves a critical role in DNA damage repair (26). Cancer cells with radioresistance highly express cyclin D1, which causes the majority of these cells to enter $\mathrm{S}$ phase, and increases radioresistance and progression (27). In the present study, rapamycin decreased the phosphorylation of S6 and the expression of cyclin D1 in NPC, which resulted in decreased cell viability, G1 phase arrest and increased DNA damage in NPC. Additionally, cells in the late G1 and G2/M phases are most sensitive to IR, whereas those in $\mathrm{S}$ phase have least radiosensitivity (28). In the present study, rapamycin arrested NPC cells in G1 phase, and therefore sensitized NPC to RT, which was supported by the observations that the combination of rapamycin and IR induced lower growth and higher DNA damage in NPC cells than either rapamycin or IR alone. These results suggested that rapamycin can retard NPC growth and improve the radiosensitivity of NPC through inhibiting both the Akt/mTOR/S6 and Akt/GSK3//cyclin D1 pro-survival signaling pathways. A recent phase I clinical trial documented the safety and tolerance of everolimus, another 
mTOR inhibitor, in the treatment of head and neck cancer when administered in combination with weekly cisplatin and intensity-modulated RT (29). As a widely used drug for suppressing autoimmune responses in organ transplantation, rapamycin is safe and may be used as a radiosensitizer in the RT of NPC. However, further in vivo studies using xenografts and immunohistochemical analysis remain to be conducted to validate the present results.

In conclusion, rapamycin improves the anti-tumor effect of IR in the treatment of NPC. The findings of the present study may facilitate further clinical investigations of combination therapy using rapamycin as a radiosensitizer to improve patient prognosis in the treatment of NPC.

\section{Acknowledgements}

The present study was supported by grants from the Science and Technology Agency of Hunan Province (grant nos. 2014SK3272 and 2016WK2035), Health Department of Hunan Province (grant nos. B2014-144 and A2016005) and Changsha Municipal Science and Technology Bureau (grant no. k1406004-61).

\section{References}

1. Low SY, Tan BS, Choo HL, Tiong KH, Khoo AS and Leong CO: Suppression of BCL-2 synergizes cisplatin sensitivity in nasopharyngeal carcinoma cells. Cancer Lett 314: 166-175, 2012.

2. Lee AW, Lin JC and Ng WT: Current management of nasopharyngeal cancer. Semin Radiat Oncol 22: 233-244, 2012.

3. Yoshizaki T, Ito M, Murono S, Wakisaka N, Kondo S and Endo K: Current understanding and management of nasopharyngeal carcinoma. Auris Nasus Larynx 39: 137-144, 2012.

4. Orlandi E, Tomatis S, Potepan P, Bossi P, Mongioj V, Carrara M, Palazzi M, Franceschini M, Bergamini C, Locati L, et al: Critical analysis of locoregional failures following intensity-modulated radiotherapy for nasopharyngeal carcinoma. Future Oncol 9: 103-114, 2013.

5. Bussink J, van der Kogel AJ and Kaanders JH: Activation of the PI3-K/AKT pathway and implications for radioresistance mechanisms in head and neck cancer. Lancet Oncol 9: 288-296, 2008.

6. Shimura T: Acquired radioresistance of cancer and the AKT/ GSK3 $\beta /$ cyclin D1 overexpression cycle. J Radiat Res 52: 539-544, 2011.

7. Hay N and Sonenberg N: Upstream and downstream of mTOR. Genes Dev 18: 1926-1945, 2004.

8. Liu Y, Chen LH, Yuan YW, Li QS, Sun AM and Guan J: Activation of AKT is associated with metastasis of nasopharyngeal carcinoma. Tumour Biol 33: 241-245, 2012.

9. Li HF, Kim JS and Waldman T: Radiation-induced Akt activation modulates radioresistance in human glioblastoma cells Radiat Oncol 4: 43, 2009

10. Xia S, Zhao Y, Yu S and Zhang M: Activated PI3K/Akt/COX-2 pathway induces resistance to radiation in human cervical cancer HeLa cells. Cancer Biother Radiopharm 25: 317-323, 2010.

11. Zhang HH, Lipovsky AI, Dibble CC, Sahin M and Manning BD: S6K1 regulates GSK3 under conditions of mTOR-dependent feedback inhibition of Akt. Mol Cell 24: 185-197, 2006.
12. Albert JM, Kim KW, Cao C and Lu B: Targeting the Akt/ mammalian target of rapamycin pathway for radiosensitization of breast cancer. Mol Cancer Ther 5: 1183-1189, 2006.

13. Shinohara ET, Cao C, Niermann K, Mu Y, Zeng F, Hallahan DE and $\mathrm{Lu} \mathrm{B}$ : Enhanced radiation damage of tumor vasculature by mTOR inhibitors. Oncogene 24: 5414-5422, 2005.

14. Cao C, Subhawong T, Albert JM, Kim KW, Geng L, Sekhar KR, Gi YJ and Lu B: Inhibition of mammalian target of rapamycin or apoptotic pathway induces autophagy and radiosensitizes PTEN null prostate cancer cells. Cancer Res 66: 10040-10047, 2006.

15. Nassim R, Mansure JJ, Chevalier S, Cury F and Kassouf W: Combining mTOR inhibition with radiation improves antitumor activity in bladder cancer cells in vitro and in vivo: A novel strategy for treatment. PLoS One 8: e65257, 2013.

16. Abraham RT and Wiederrecht GJ: Immunopharmacology of rapamycin. Annu Rev Immunol 14: 483-510, 1996.

17. Prevo R, Deutsch E, Sampson O, Diplexcito J, Cengel K, Harper J, O'Neill P, McKenna WG, Patel S and Bernhard EJ: Class I PI3 kinase inhibition by the pyridinylfuranopyrimidine inhibitor PI-103 enhances tumor radiosensitivity. Cancer Res 68: 5915-5923, 2008.

18. Suárez C, Rodrigo JP, Rinaldo A, Langendijk JA, Shaha AR and Ferlito A: Current treatment options for recurrent nasopharyngeal cancer. Eur Arch Otorhinolaryngol 267: 1811-1824, 2010.

19. LoPiccolo J, Blumenthal GM, Bernstein WB and Dennis PA: Targeting the PI3K/Akt/mTOR pathway: Effective combinations and clinical considerations. Drug Resist Updat 11: 32-50, 2008.

20. Kim MK, Kim TJ, Sung CO, Choi CH, Lee JW, Kim BG and Bae DS: High expression of mTOR is associated with radiation resistance in cervical cancer. J Gynecol Oncol 21: 181-185, 2010.

21. Romano G: The role of the dysfunctional akt-related pathway in cancer: Establishment and maintenance of a malignant cell phenotype, resistance to therapy, and future strategies for drug development. Scientifica (Cairo) 2013: 317186, 2013.

22. Liu SC, Tsang NM, Chiang WC, Chang KP, Hsueh C, Liang Y, Juang JL, Chow KP and Chang YS: Leukemia inhibitory factor promotes nasopharyngeal carcinoma progression and radioresistance. J Clin Invest 123: 5269-5283, 2013.

23. Chung J, Kuo CJ, Crabtree GR and Blenis J: Rapamycin-FKBP specifically blocks growth-dependent activation of and signaling by the 70 kd S6 protein kinases. Cell 69: 1227-1236, 1992.

24. Terada N, Takase K, Papst P, Nairn AC and Gelfand EW: Rapamycin inhibits ribosomal protein synthesis and induces G1 prolongation in mitogen-activated $\mathrm{T}$ lymphocytes. J Immunol 155: 3418-3426, 1995.

25. Baldin V, Lukas J, Marcote MJ, Pagano M and Draetta G: Cyclin $\mathrm{D} 1$ is a nuclear protein required for cell cycle progression in G1. Genes Dev 7: 812-821, 1993.

26. Jirawatnotai S, Hu Y, Michowski W, Elias JE, Becks L, Bienvenu $\mathrm{F}$, Zagozdzon A, Goswami T, Wang YE, Clark AB, et al: A function for cyclin D1 in DNA repair uncovered by protein interactome analyses in human cancers. Nature 474: 230-234, 2011.

27. Shimura T, Fukumoto M and Kunugita N: The role of cyclin $\mathrm{D} 1$ in response to long-term exposure to ionizing radiation. Cell Cycle 12: 2738-2743, 2013.

28. Pawlik TM and Keyomarsi K: Role of cell cycle in mediating sensitivity to radiotherapy. Int J Radiat Oncol Biol Phys 59: 928-942, 2004

29. Fury MG, Lee NY, Sherman E, Ho AL, Rao S, Heguy A, Shen R, Korte S, Lisa D, Ganly I, et al: A phase I study of everolimus + weekly cisplatin + intensity modulated radiation therapy in head-and-neck cancer. Int J Radiat Oncol Biol Phys 87: 479-486, 2013. 